\title{
Perceived stress before and during the COVID-19 pandemic
}

Dr Mark Ward and Professor Rose Anne Kenny

The Irish Longitudinal Study on Ageing (TILDA)

\section{December 2020}

Copyright () The Irish Longitudinal Study on Ageing 2020

The Irish Longitudinal Study on Ageing

Trinity College Dublin

Dublin 2

Tel: +35318962509

Email: tilda@tcd.ie

Website: www.tilda.ie

https://www.doi.org/10.38018/TildaRb.2020-03 


\section{Introduction}

Perceived stress is associated with physical and mental health, and cognitive decline. There has been some research published on the effect of the COVID-19 pandemic on perceived stress among healthcare workers and COVID-19 patient, but it is not yet known how the pandemic has affected the mood of the general community-dwelling population over 60 years. We hypothesise that concerns about contracting the COVID-19 virus may be heightened among older adults as they are more susceptible to being infected by the virus, suffer serious consequences from infection, and have the highest risk of mortality. These fears may manifest themselves in heightened levels of stress. Furthermore, the many changes people have had to make to their normal daily lives, such as being confined to the home, and avoiding social contacts, are also likely to increase feelings of stress.

\section{Method}

This research brief is based on 3,614 Self-Completion Questionnaires completed by participants of The Irish Longitudinal Study on Ageing (TILDA). This study population consists of community-dwelling adults aged 60 years and older. Data were collected between June and October 2020.

Stress over the previous month was measured using the 4-item version of the Perceived Stress Scale (PSS-4; Cohen et al., 1983). The PSS captures how unpredictable, uncontrollable, and overloaded a respondent's life is, and is a measure of chronic stress associated with generalised stress perception. The PSS-4 includes four questions:

How often have you felt that you were unable to control the important things in your life?

How often have you felt confident about your ability to handle your personal problems?

How often have you felt that things were going your way?

How often have you felt difficulties were piling up so high that you could not overcome them?

Each question is answered on 5-point Likert scale that ranges from 0 (never) to 4 (very often). Scores range from 0 to 16 , with higher scores indicating greater stress. 
TILDA has included this measure of stress at each of five waves of data collection conducted at 2-year intervals since 2009. We are therefore in the unique position of being able to examine differences in stress levels before and during the pandemic. In this brief report, we describe changes in stress, measured using the PSS-4, over a two-year period using data from the fifth wave of TILDA, carried out in 2018/19, and information provided by TILDA participants who completed the COVID-19 selfcompletion questionnaire between June and October 2020. We also describe the changes in perceived stress among different groups.

\section{Results}

Overall, the average perceived stress score increased significantly between 2018/2019 and March to October 2020. Over this two-year period, the average score among TILDA participants increased by almost $20 \%$, from 4.0 to 4.7 . Figure 1 shows that both men and women experienced a significant increase in perceived stress since the start of the COVID-19 pandemic in March 2020. Women reported higher levels of perceived stress at both time points but the difference between men and women is larger now than it was before the COVID-19 pandemic.

Figure 1. Differences in average perceived stress before and during the COVID-19 pandemic among men and women

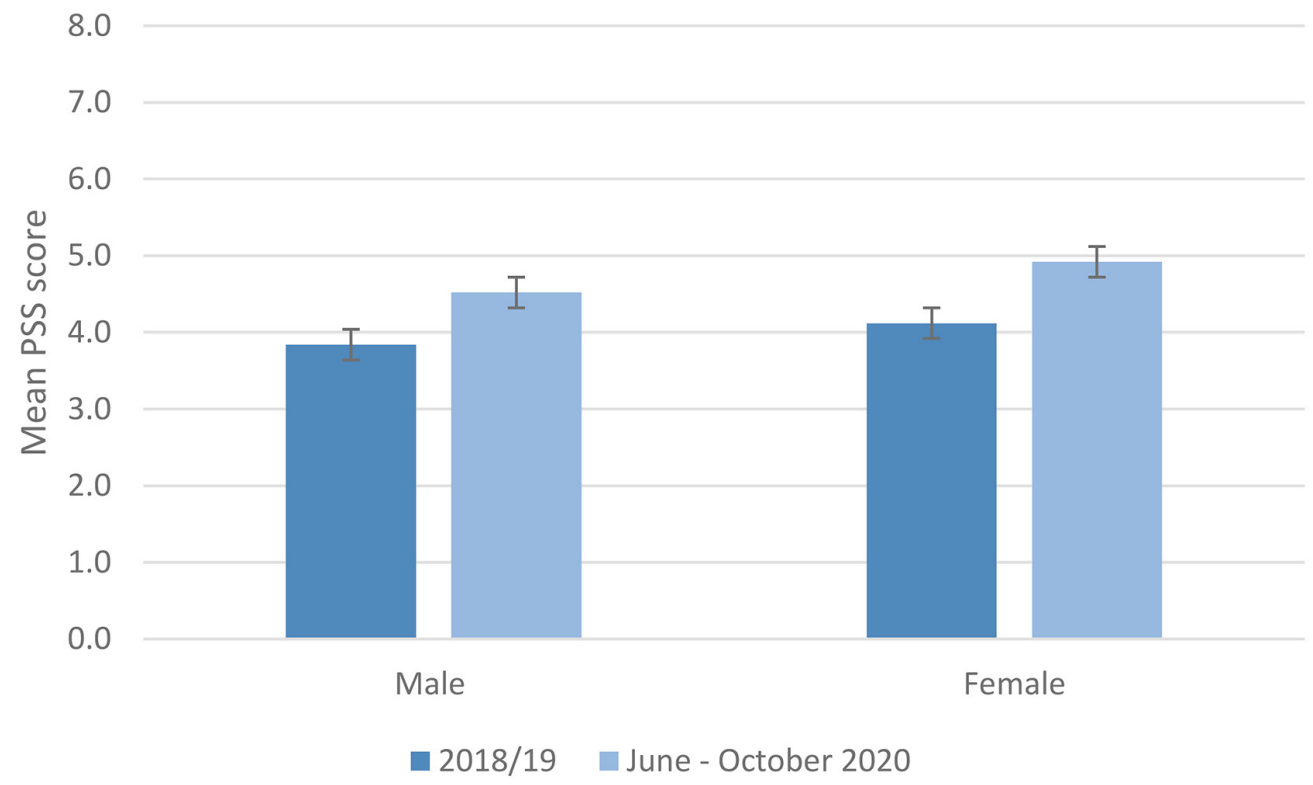

Whether or not people live alone or in shared accommodation is likely to affect perceived stress among older adults. Many of the opportunities people who live alone have for social contacts with family and friends are now denied to them while those who live with others may experience increased stress due being confined with 
others. As shown in Figure 2, there was no difference in perceived stress according to whether men or women lived alone or with others. There was also no difference in perceived stress before and after the start of the pandemic among either men or women who live alone. Perceived stress did however increase among both men and women who lived with others. The change was larger among women, with average perceived stress scores increasing from 4.0 pre COVID-19 to 5.0 since the beginning of the pandemic.

Figure 2. Differences in average perceived stress before and during the COVID-19 pandemic by gender and living alone or with others

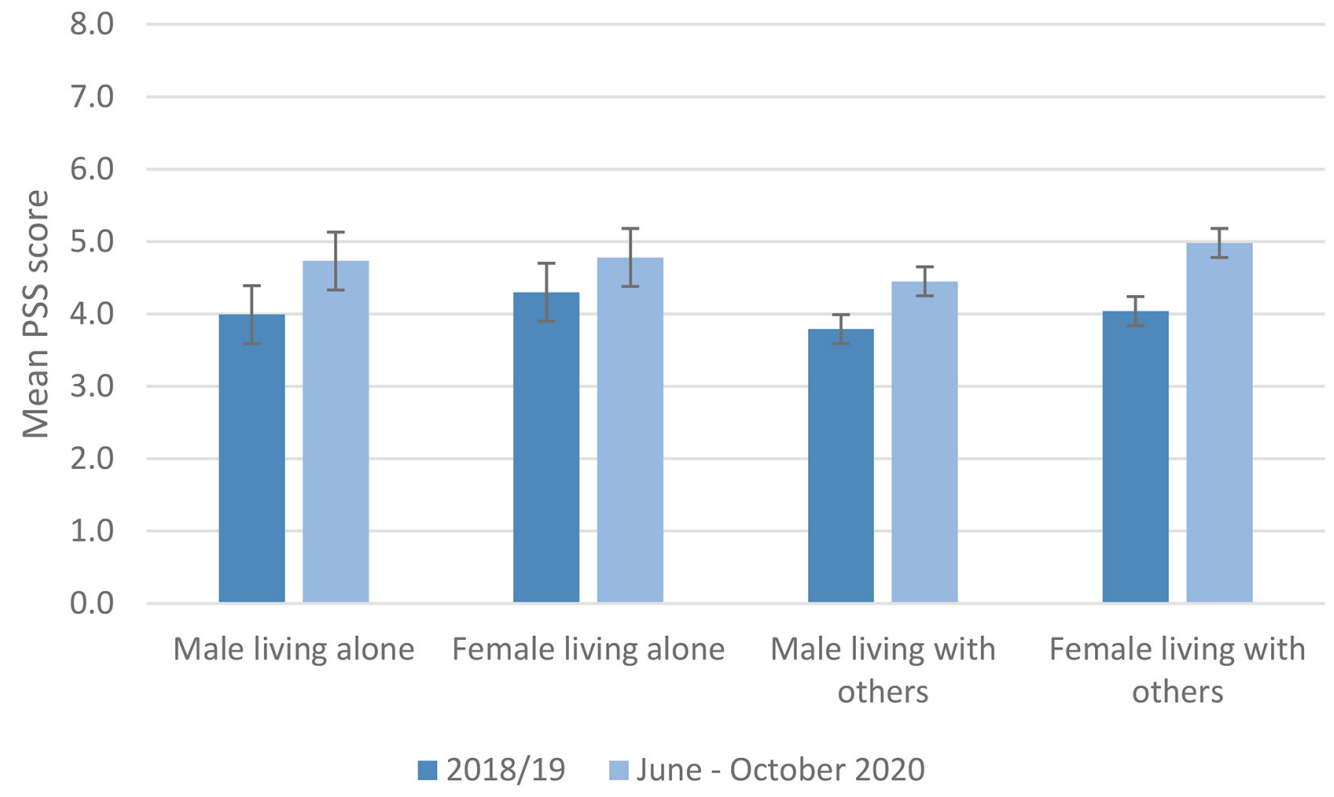

Perceived stress increased significantly among both men and women aged 70 years and older as well as women aged less than 70 years (Figure 3). There was no increase in perceived stress among men under the age of 70. 
Figure 3. Differences in average perceived stress before and during the COVID-19 pandemic among men and women under and over 70 years of age

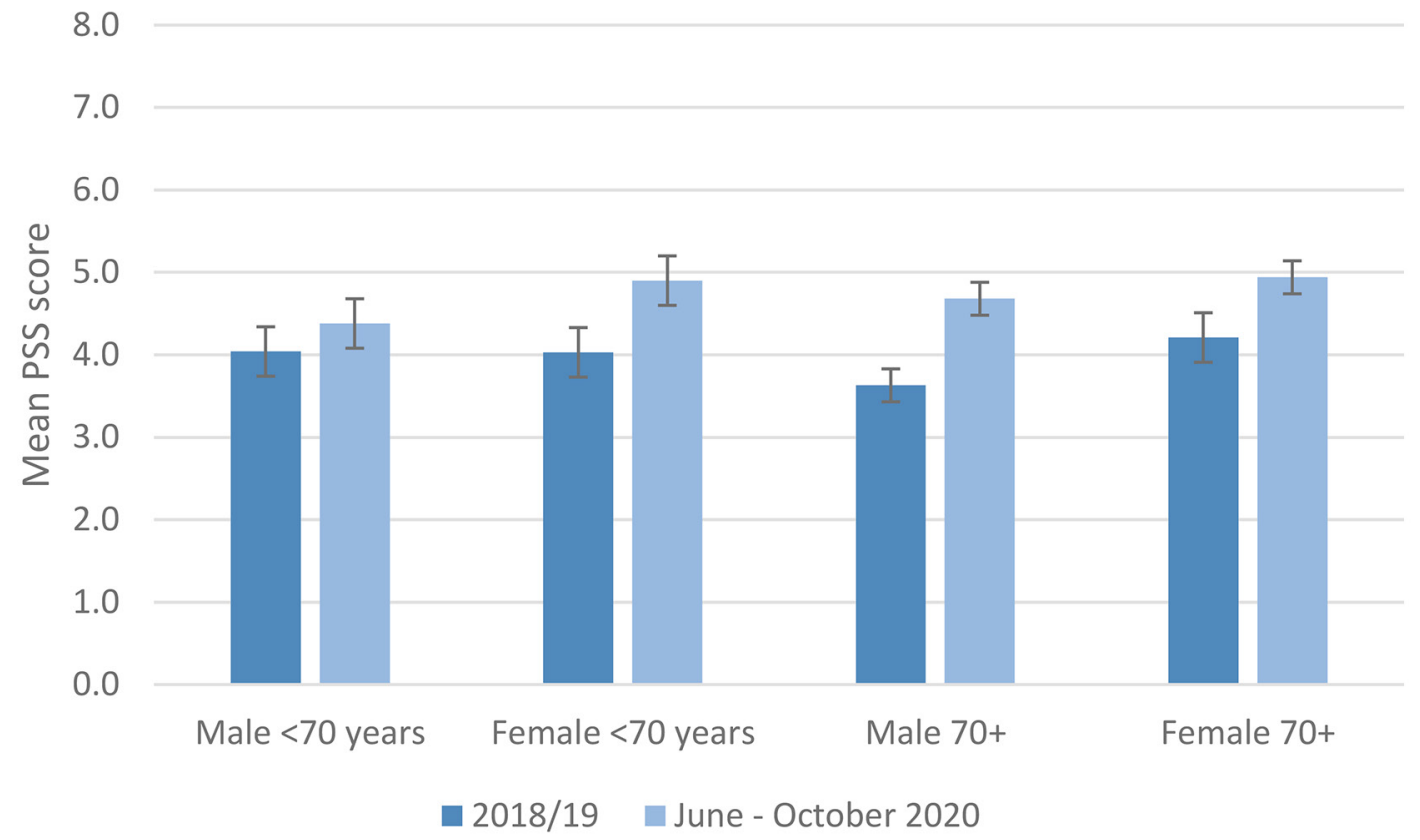

As shown in Figure 4, levels of perceived stress were similar among older adults in rural and urban locations, and stress has increased among both groups since the start of the COVID-19 pandemic. 
Figure 4. Differences in average perceived stress before and during the COVID-19 pandemic by urban and rural location

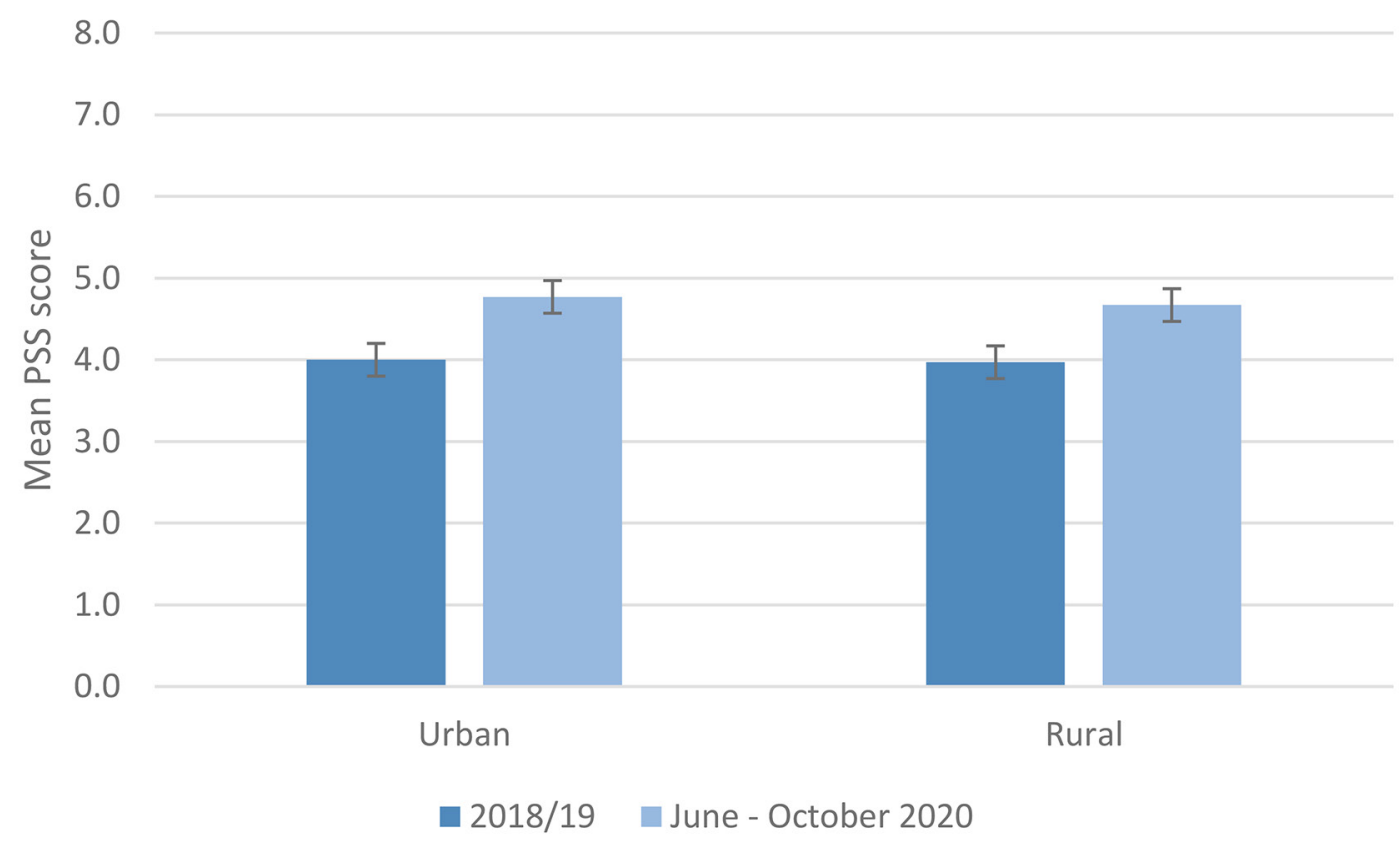

\section{Conclusion}

Since the beginning of the COVID-19 pandemic, perceived stress has increased significantly among older adults. This increase will likely negatively affect both physical and psychological wellbeing among this population. Older adults who live in shared households experienced the greatest increase in stress. At this early stage, we suspect that this may be driven by fear of contracting the COVID-19 virus from other household members who may not be confined to home. This may also manifest itself in a perceived lack of control of ones' own environment and wellbeing. Alternatively, levels of stress may have increased among this group due to the pressures of being confined with others in a situation where usual activities and behaviours have been changed dramatically. Combining the rich existing TILDA data source with new data collected during the pandemic will enable us to investigate the reasons behind the results reported here.

\section{References}

Cohen, S., Kamarck, T., \& Mermelstein, R. (1983). A global measure of perceived stress. Journal of Health and Social Behavior, 385-396 